\title{
Faturamento de novos produtos na indústria do estado de São Paulo: análise dos fatores de influência
}

\author{
New product revenue in São Paulo state industry: analysis of \\ influencing factors
}

\author{
Antonio Carlos Pacagnella Junior ${ }^{1}$ \\ Sergio Kannebley Júnior ${ }^{2}$ \\ Sérgio Luís da Silva ${ }^{3}$ \\ Alexandre Pereira Salgado Junior ${ }^{4}$ \\ Geciane Silveira Porto ${ }^{5}$
}

\begin{abstract}
Resumo: Diversos estudos têm sido realizados no intuito de analisar os impactos da inovação tecnológica no desempenho comercial das empresas. Com isso, este estudo visa analisar os fatores de influência sobre o percentual do faturamento com a venda de novos produtos na indústria do Estado de São Paulo. Trata-se de uma pesquisa com características descritivas e explicativas, cuja abordagem é quantitativa, utilizando dados secundários provindos da Pesquisa de Atividade Econômica Paulista (PAEP), realizada pela Fundação SEADE. Os resultados mostram que os fatores de influência sobre o faturamento provindo de novos produtos são: a origem do capital controlador; a empresa pertencer a um grupo empresarial; o total de pessoal em pesquisa e desenvolvimento; o pessoal alocado diretamente na produção; as fontes de informação para atividades inovativas; e o apoio governamental para a inovação tecnológica.

Palavras-chave: Faturamento provindo de novos produtos. Indústria paulista. Inovação tecnológica. Regressão linear múltipla. Indicadores de inovação.
\end{abstract}

\begin{abstract}
Several studies have been conducted to analyze the impacts of technological innovation on the trade performance of enterprises. Therefore, the main objective of this study is to investigate the factors that influence the percentage of sales revenue derived from new products in São Paulo State industry. This is a descriptive and explanatory research that uses a quantitative approach. It used secondary data from the PAEP, Survey of Economic Activity in the State of São Paulo, which was conducted by SEADE (State Data Analysis System foundation). The results obtained show that the factors that influence new product revenue are: controlling shareholder nationality, being part of an entrepreneurial group, the number of people involved in research and development, the number of employees working directly in manufacturing operations, information sources for innovation activities, and the government support for technological innovation.
\end{abstract}

Keywords: Revenue derived from new products. São Paulo State industry. Technological innovation. Multiple linear regression. Innovation indicators.

\section{Introdução}

Em decorrência das constantes mudanças sociais, políticas e, principalmente, econômicas, características da globalização, as empresas têm operado em ambientes altamente competitivos, visando atender aos mercados cada vez mais exigentes.

Neste contexto, a empresa contemporânea deve estar orientada a introduzir continuamente novas ideias ao seu portfólio de bens e serviços, gerenciando, assim, sua capacidade de inovação de forma que esta contribua cada vez mais para a obtenção de seus resultados.

\footnotetext{
${ }^{1}$ Programa de Pós-graduação em Engenharia de Produção, Universidade Metodista de Piracicaba - UNIMEP, Rod. Luis Ometto, Km 24, SP 306, CEP 13451-900, Santa Bárbara d’Oeste, SP, Brasil, e-mail: acpjr1@gmail.com

${ }^{2}$ Departamento de Economia, Faculdade de Economia Administração e Contabilidade de Ribeirão Preto - FEA-RP/USP, Av. dos Bandeirantes, 3900, Sala 42, Bloco C, CEP 14040-900, Ribeirão Preto, SP, Brasil, e-mail:skj@usp.br

${ }^{3}$ Programa de Pós-graduação em Engenharia de Produção, Departamento de Ciência da Informação, Universidade Federal de São Carlos - UFSCar, Rod. Washington Luiz, Km 235, Monjolinho, CEP 13565-905, São Carlos, SP, Brasil, e-mail: sergiol@ufscar.br

${ }^{4}$ Faculdade de Economia Administração e Contabilidade de Ribeirão Preto - FEA-RP/USP, Av. dos Bandeirantes, 3900, Sala 58, Bloco A, CEP 14040-900, Ribeirão Preto, SP, Brasil, e-mail: asalgado@usp.br

${ }^{5}$ Departamento de Administração, Faculdade de Economia Administração e Contabilidade de Ribeirão Preto - FEA-RP/USP, Av. dos Bandeirantes, 3900, Sala 40, Bloco C, CEP 14040-900, Ribeirão Preto, SP, Brasil, e-mail:geciane@usp.br
} 
As pesquisas sobre inovação tecnológica e temas relacionados justificam-se por diversas razões, dentre elas, destacam-se as propostas por Viotti e Macedo (2001). Primeiramente há a razão científica, baseada na ideia de que estudar a inovação tecnológica pode contribuir para o entendimento de questões referentes à dinâmica da ciência e tecnologia, tais como, os impactos do avanço tecnológico na sociedade, economia, emprego, na qualidade de vida e no meio ambiente.

Em segundo, há a chamada razão política, baseada na identificação das necessidades científicas que podem levar à elaboração de políticas públicas mais efetivas, o que, conforme Archibugi, Howells e Michie (1999), pode permitir a um país obter vantagem no mundo globalizado.

Por último, tem-se a razão pragmática, relacionada à identificação de oportunidades tecnológicas e fundamentação de decisões de investimento, fornecendo subsídios para a elaboração das estratégias tecnológicas realizadas pelas empresas.

A escolha da indústria paulista para a análise proposta neste trabalho justifica-se tanto pela contribuição expressiva do Estado para a economia nacional (cerca de $40 \%$ do Produto Interno Bruto) como pela importância tecnológica no âmbito nacional. Isso é evidente ao se verificar os dados da Pesquisa de Inovação Tecnológica (PINTEC) realizada pelo Instituto Brasileiro de Geografia e Estatística (IBGE), referente ao ano de 2005, da qual se destacam:

- O Estado possui cerca de 35,33\% das empresas inovadoras (produto ou processo) do total nacional;

- Cerca de 55,67\% do total de recursos humanos alocados em pesquisa e desenvolvimento estão nas organizações paulistas; e

- Dos dispêndios em atividades inovativas nas empresas nacionais, 55,47\% são investidos no Estado de São Paulo.

Ao se observar os fatores supracitados, é possível concluir que a realização de estudos relacionados à inovação tecnológica que permitam entender de forma mais profunda as dinâmicas relacionadas a este fenômeno nas empresas paulistas é de suma importância para a economia e evolução tecnológica nacional.

Para a realização do presente estudo, foi adotado, como indicador, o percentual de faturamento com novos produtos, que reflete o impacto da inovação tecnológica nos resultados comerciais obtidos pelas empresas industriais de São Paulo.

Desta forma, o objetivo principal deste trabalho é analisar os fatores de influência neste indicador, utilizando os dados da Pesquisa de Atividade Econômica Paulista (PAEP), referente ao período de 1999 a 2001.
Para atingir o objetivo principal proposto, foram selecionados diversos fatores que possivelmente impactam o fenômeno, selecionados a partir de estudos anteriores de diversos autores sobre a inovação tecnológica, que serão tratados na próxima seção. Entre eles estão: o tamanho das empresas; a orientação exportadora; a origem do capital controlador; o salário médio; a idade da empresa; o fato da empresa pertencer a um grupo empresarial; o percentual de pessoas alocadas diretamente na produção; a origem da maior parte do faturamento da empresa (se de bens ou serviços); os investimentos financeiros e de recursos humanos em P\&D; a realização de cooperação em $P \& D$; a presença de laboratório ou departamento específico de P\&D; o apoio governamental para atividades inovativas; a atribuição de importância para fontes de informação para atividades inovativas; e a ampliação ou a redução do portfólio de bens e serviços.

O trabalho está dividido como segue: a primeira seção é a introdução; a segunda seção apresenta um referencial teórico sobre inovação tecnológica e sobre o percentual de faturamento provindo de novos produtos; a terceira seção traz os aspectos metodológicos do trabalho; a quarta seção descreve a amostra utilizada; a quinta seção apresenta os resultados encontrados; e, na última seção, são feitas as considerações finais.

\section{Inovação tecnológica e desempenho empresarial}

Por estar posicionada no cerne da mudança econômica, a inovação tecnológica é um fenômeno amplamente discutido no meio acadêmico e, por isso, pode ser definida de várias formas. Uma das principais definições sobre o tema é de Schumpeter (1991), que, na metade do século XX, propôs a inovação tecnológica como a introdução de um novo produto ou a mudança em algum produto já existente, um novo processo de produção em uma determinada indústria, a abertura de novos mercados, a conquista de novas fontes de suprimento de matéria-prima e a reestruturação organizacional.

Damanpour e Schneider (2006) definem a inovação como a introdução no mercado de um novo bem ou serviço, a adoção de uma nova tecnologia, uma nova estrutura organizacional ou um novo sistema administrativo.

Já Francis e Bessant (2005), classificam a inovação tecnológica em quatro categorias: a inovação em produtos, em processos, em posicionamento, que consiste em definir ou redefinir o posicionamento de uma empresa ou de um produto no mercado e a inovação de paradigma que consiste em definir ou redefinir os paradigmas da organização, podendo ser internos (valores organizacionais e políticas 
de tomada de decisão) e externos (o modelo de negócios, a forma conceitual que a empresa utiliza para prosperar no mercado).

Embora existam várias perspectivas para se observar a inovação tecnológica, uma das mais relevantes para estudos empíricos como este é a do Manual de OSLO, pois ele é a principal fonte internacional de diretrizes para coleta e uso de dados sobre atividades inovadoras da indústria, produzido pela Organização para a Cooperação e Desenvolvimento Econômico (OCDE). A inovação tecnológica é por ela definida como a implementação de um produto (bem ou serviço) novo ou significativamente melhorado; um novo processo; um novo método de marketing; ou ainda, um novo método organizacional relativo a práticas de negócios, na organização do local de trabalho ou nas suas relações externas (ORGANISATION..., 2005).

De acordo com Dosi (1988), a inovação tecnológica origina-se da necessidade de solução de um problema tecnológico em que somente os conhecimentos disponíveis, até o momento, não são suficientes para resolver; além disso, o autor ressalva que esta solução deve considerar critérios de custos e comercialidade, as capacidades específicas dos inventores e novas descobertas e criações.

Desta forma, como ilustrado na Figura 1, um problema tecnológico demanda a necessidade ou gera a oportunidade para que aconteça a inovação tecnológica.

A dinâmica deste processo, segundo afirmam Nuchera, Serrano e Morote (2002), acontece principalmente na forma market-pull, na qual o mercado é o principal elemento desencadeante do processo de inovação tecnológica, ou seja, o gerador dos problemas tecnológicos.

Carayannis e Roy (2000) argumentam que a tecnologia é entendida como uma espécie de mercadoria que seria produzida para atender a uma demanda, assim, a inovação tecnológica aconteceria como resposta a uma necessidade de mercado previamente detectada, ou uma resposta dos agentes desenvolvedores de tecnologia a uma demanda de mercado (feedback tecnológico). Os autores propõem ainda que esta resposta realimenta o sistema gerando novas necessidades (ou problemas tecnológicos) que devem ser atendidas sucessivamente.

Sobre o processo de inovação e a sua respectiva gestão, são encontradas na literatura diversas propostas, desde a inicial, o chamado "modelo linear de inovação" (ou Science-push) de Bush (1945), passando pelo modelo de elo de cadeia de Kline e Rosenberg (1986), no qual a inovação tecnológica é o resultado de interações entre oportunidades de mercado e a base de competências por parte das empresas, sendo composta por diversos subprocessos como invenção, desenvolvimento e comercialização, porém sem uma sequência claramente definida e com ocorrência de realimentações entre os subprocessos e culminando no modelo Open Innovation. Este se baseia na utilização de caminhos internos ou externos, com o envolvimento de outras organizações que trocam experiências contribuindo para o sucesso do processo inovativo.

Introduzir inovações tecnológicas, de acordo com Millson e Wilemon (2006), é de suma importância para a competitividade empresarial e, neste contexto, torna-se fundamental que os aspectos relevantes do fenômeno para o meio empresarial sejam mensurados pelo uso de indicadores.

Sendo assim, diversos indicadores têm sido utilizados para analisar o fenômeno da inovação no meio empresarial, sejam eles relativos ao esforço inovador (investimentos de recursos financeiros em P\&D, recursos humanos alocados em P\&D e outros), ou ao de desempenho inovador (obtenção de patentes, percentual de faturamento provindo da venda de novos produtos e etc.).

De acordo com Shapiro (2006), devido à complexidade da inovação tecnológica, mensurá-la com apenas um tipo de indicador é uma tarefa árdua. Entretanto, no meio empresarial, o percentual de faturamento provindo da venda de novos produtos é especificamente um indicador muito útil, pois permite analisar os impactos das inovações de produto sobre a composição geral do faturamento, revelando

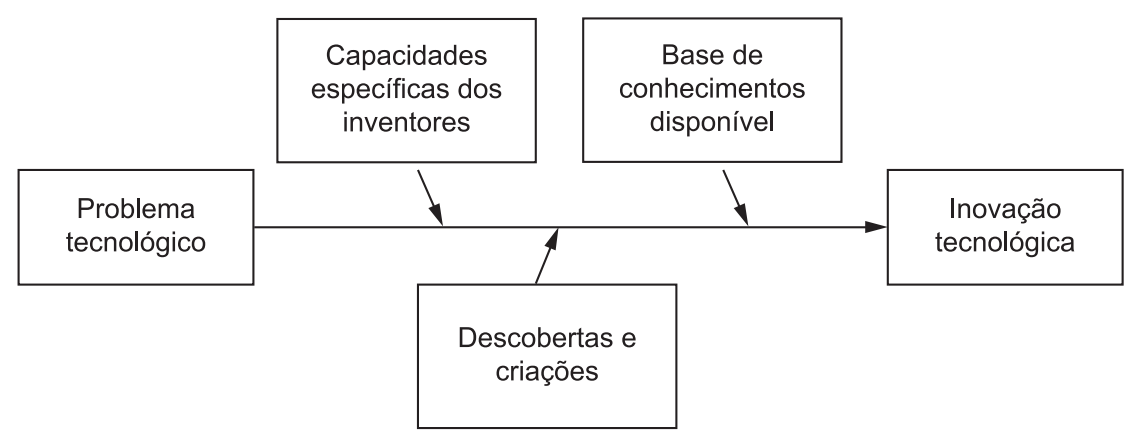

Figura 1. Problema tecnológico levando à inovação tecnológica segundo Dosi (1988). Fonte: Adaptado de Dosi (1988). 
informações importantes como o grau de inovatividade da empresa e a performance da inovação no mercado.

Dessa forma, o faturamento provindo da venda de produtos novos constitui um indicador sobre como o desempenho empresarial pode ser impactado pela introdução de inovações tecnológicas.

É preciso destacar que diversos fatores podem influenciar sua obtenção e resultados empresariais tais como o indicador citado, as características do mercado em que a empresa atua, suas ações de marketing, a qualidade do novo produto e outros, gerando análises sob diversas perspectivas (BAKER; SINKULA, 2005).

Porém, quando o tema é tratado pela perspectiva da inovação tecnológica, a busca pela identificação e análise dos fatores que influenciam os diversos aspectos do desempenho inovativo das empresas é tema de grande interesse no meio acadêmico, gerando uma extensa literatura sobre o assunto. Isso se dá pela importância que a compreensão mais profunda das dinâmicas inovativas possui para que decisões de investimento sejam tomadas e estratégias tecnológicas sejam traçadas.

Nesse contexto, a literatura sobre o assunto apresenta diversos estudos que indicam a presença de uma relação positiva entre a inovação tecnológica e o desempenho empresarial, destacando-se os estudos de Freel e Robson (2004), Hall e Bagchi-Sem (2002) e Mansury e Love (2008).

Assim, cabe destacar que, para estabelecer um referencial teórico-empírico adequado para a análise realizada neste trabalho, tomou-se como base o trabalho dos seguintes autores (cujos resultados suportaram a escolha das variáveis do modelo conceitual apresentado na Figura 2):

- Cooper, Edgett e Kleinshmidt (2005) e Milson e Wilemon (2006), que analisaram a influência de fatores como a infraestrutura organizacional, a experiência da empresa, os recursos investidos em P\&D e a gestão de portfólio no sucesso comercial de novos produtos;

- Kannebley Júnior, Porto e Pazzelo (2005), cujos resultados apontam para a influência da orientação exportadora, a origem do capital controlador e do tamanho da empresa no desempenho inovador;

- Quadros et al. (2001) e Frishammar e Hörte (2005), que analisaram a influência das fontes de informação para as atividades inovativas e sua importância para os resultados em termos de inovação;

- Hagedoorn (2002), que destaca a importância da cooperação em $\mathrm{P} \& \mathrm{D}$ para o desempenho inovador;

- Shefer e Fenkel (2005), que destacam a idade, o tamanho e a orientação exportadora da empresa, como características que influenciam a inovação tecnológica;

- Sharp e Pavitt (1993), Fonseca (2001) e Freitas e Tunzelmann (2008), cujos trabalhos ressaltam a relevância do apoio governamental para o desempenho inovativo das firmas;

\section{Variáveis independentes}

- Investimento de recursos financeiros em P\&D

- Investimento de recursos humanos em P\&D

- Presença de laboratório ou departamento específico de P\&D

- Cooperação em P\&D

- Apoio governamental para atividades de inovação

- Fontes de informação para inovação

- Tamanho da empresa

- Orientação exportadora

- Origem do capital controlador

- Média salarial

- Recursos humanos ligados diretamente à produção

- Fazer parte de grupo empresarial

- Idade da empresa

- Receita provinda na maior parte de bens ou serviços industriais

- Ampliar variedade de produtos oferecidos

Figura 2. Modelo conceitual de pesquisa. Fonte: Elaborado pelos autores.

Variável dependente

- Percentual do faturamento provindo de novos produtos 
- Mahmood e Lee (2004) e Hsieh, Yeh e Chen (2010), que ressaltam a influência da organização pertencer a um grupo empresarial em sua dinâmica inovativa; e

- Castellacci (2008), cujo trabalho destaca as diferenças nos padrões inovativos de empresas industriais e prestadoras de serviço.

Sendo assim, o presente estudo, alinhando-se aos trabalhos supracitados, aborda a obtenção de faturamento provindo de novos produtos nas empresas industriais paulistas, propondo para este fim um modelo conceitual que envolve os principais fatores citados na literatura sobre inovação, de forma a promover uma investigação sistêmica de cunho empírico sobre o tema estudado.

\section{Aspectos metodológicos}

O presente trabalho possui caráter descritivo e explicativo, buscando analisar as influências na obtenção do faturamento provindo de novos produtos por parte das empresas industriais paulistas por meio de uma abordagem quantitativa. Para tanto, foram utilizados dados secundários provindos da Pesquisa de Atividade Econômica Paulista (PAEP), realizada no ano de 2002 e que abrange o período que vai de 1999 a 2001, sua versão mais atual.

Para a determinação da amostra, a Fundação SEADE utilizou o Cadastro de Empresas (CEMPRE), fornecido pelo Instituto Brasileiro de Geografia e Estatística (IBGE), no qual foram selecionados 1.006.037 registros, que, por sua vez, deu origem a uma amostra de 42.023 empresas industriais.

A amostragem realizada pela PAEP se deu de maneira estratificada, que, de acordo com Malhotra (2001), é um processo de dois estágios em que a população é dividida em subpopulações ou estratos, que correspondem neste caso a 21 setores industriais para os quais a pesquisa apresenta significância estatística além da indústria de forma agregada.

A escolha pela base de dados da PAEP pode ser justificada por dois motivos: o primeiro é relativo a seu tamanho e por ter sido especificamente desenhada para colher dados da indústria paulista; e o segundo é relativo a esta base ter sido relativamente pouco explorada por estudos empíricos em comparação com outros bancos de dados como a Pesquisa de Inovação Tecnológica (PINTEC) do Instituto Brasileiro de Geografia e Estatística (IBGE) e a pesquisa realizada pela Associação Nacional de Pesquisa e Desenvolvimento das Empresas Inovadoras (ANPEI).

\subsection{Técnica de análise de dados}

Neste trabalho, a técnica de análise utilizada foi a regressão linear múltipla, que, segundo Hair et al. (1998), pode ser definida como uma técnica estatística multivariada que deve ser utilizada quando o objetivo do pesquisador é analisar os efeitos de variáveis independentes, que também podem ser chamadas de preditoras ou de variáveis explicativas, sobre uma variável dependente, chamada de variável de resposta.

Para Johnson e Wichern (1992), o objetivo desta técnica é desenvolver uma equação que permita ao pesquisador prever ou explicar a variável de resposta, tomando valores fornecidos para as variáveis independentes, podendo se obter como modelo de regressão a seguinte equação:

$$
y=\beta_{0}+\beta_{1} x_{1}+\beta_{2} x_{2}+\beta_{3} x_{3}+\ldots+\beta_{n} x_{n}+\varepsilon
$$

De acordo com Greene (1993), a técnica de regressão linear múltipla exige que sejam feitas algumas suposições, que são descritas abaixo:

- Linearidade: as relações entre as variáveis preditoras e as variáveis de resposta são lineares;

- $\mathrm{E}\left[\varepsilon_{\mathrm{i}}, \mathrm{X}_{\mathrm{i}}\right]=0, \mathrm{X}$ não estocástico: o erro não é correlacionado com as variáveis preditoras; e

- $\mathrm{E}\left[\varepsilon_{\mathrm{i}}\right]=0$, o valor esperado dos resíduos é zero. Segundo Maddala (1992), a técnica em questão é adequada quando o pesquisador busca explicar uma variável dependente métrica, entretanto as variáveis dependentes que compõem o modelo de regressão podem ser métricas ou binárias, ou dummies, variáveis que possuem somente dois estados, assumindo valor igual a zero, quando o efeito da variável não está presente, e valor igual a um, quando este efeito está presente.

Um ponto fundamental sobre a aplicação da técnica de regressão linear múltipla é a acuracidade do modelo de regressão, que pode ser aferida, de acordo com Maddala (1992), utilizando-se o coeficiente de determinação $\left(\mathrm{R}^{2}\right)$, que pode ser definido como a proporção da variância da variável dependente que é explicada pelas variáveis independentes. $\mathrm{O}$ valor deste coeficiente pode variar entre 0 e 1 , sendo que, quanto mais próximo de 1 , maior o poder explicativo do modelo de regressão.

Outro aspecto relevante da técnica de regressão múltipla é a importância da verificação da multicolinearidade entre as variáveis dependentes o que reduz o poder explicativo do modelo. Para esta avaliação, Garson (2008) sugere a utilização do Variance Inflation Factor (VIF), que avalia o aumento da variância devido à presença de multicolinearidade e é calculado pela Equação 1:

$$
V I F=\frac{1}{1-R^{2}}
$$

Segundo Gujarati (2000), o limite do VIF para estabelecer se uma variável não é colinear é 4, sendo que, se este valor for superior a 10, a variável é altamente colinear. 


\subsection{Modelo de pesquisa}

Para atingir os objetivos deste trabalho, utilizando-se a técnica de regressão múltipla, inicialmente foi proposto um modelo conceitual que foi construído com o suporte dos trabalhos citados no final da seção 2 .

Cabe ressaltar que a seleção das variáveis que compõem o modelo foi realizada com base no exposto nestes trabalhos e em sua disponibilidade no banco de dados adotado neste trabalho, sendo ampla, porém não exaustiva (Figura 2).

\section{Descrição da amostra}

A amostra utilizada na PAEP foi construída de forma a garantir significância estatística para a indústria paulista de forma agregada e para vinte e um estratos que representam setores industriais.
A Figura 3 apresenta a contribuição percentual de cada setor para a amostra e também uma comparação sobre o percentual de empresas que obtiveram faturamento com a venda de novos produtos.

Para melhor compreender os dados apresentados na Figura 3, é preciso ressaltar que a quantidade de empresas presentes em cada estrato é diferente, sendo pequena em alguns casos, como no estrato referente à Indústria Extrativa e em Outras Indústrias, que apresentam de forma aglutinada algumas divisões para garantir a significância estatística.

Ao analisar a composição geral da amostra, é possível perceber que os setores que apresentam maior participação são os de Produtos Metálicos com $11,98 \%$ do total, seguido pelos setores de Vestuário e Acessórios com 11,49\%, Outras Indústrias com $11,03 \%$, Alimentos e Bebidas com $9,92 \%$ e o setor de Máquinas e Equipamentos com 7,65\% do total.

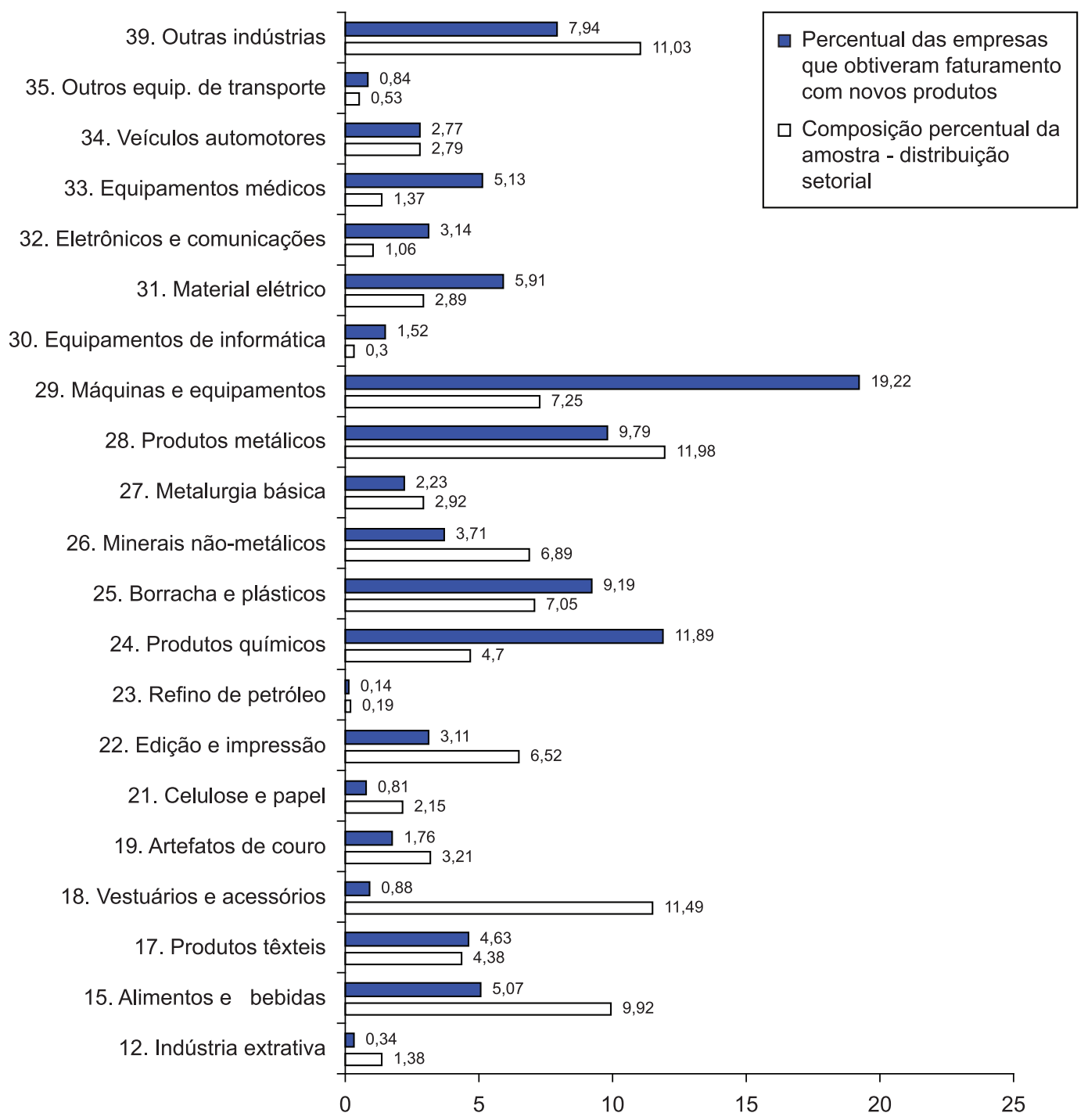

Figura 3. Composição setorial da amostra. 
Os setores com menor participação nesta composição são os setores de Refino de Petróleo com $0,2 \%$ do total, Equipamentos de Informática com 0,3\%, Outros Equipamentos de Transporte com $0,53 \%$, Equipamentos Médicos com 1,37\% e a Indústria Extrativa com apenas 1,38\% do total de empresas da amostra.

Quando se observam os dados referentes ao faturamento provindo de novos produtos, percebe-se em primeira instância o baixo percentual de empresas, sendo no total apenas 7,04\% da amostra. É relevante destacar que entre os setores que mais possuem empresas industriais que obtiveram faturamento com novos produtos está o setor de Máquinas e Equipamentos com 19,22\% do total, além dos setores de Produtos Químicos que correspondem a apenas $4,70 \%$ do total da amostra, mas em que estão $11,89 \%$ das empresas que faturaram com novos produtos e o setor de Equipamentos Médicos que, embora corresponda a apenas a $1,37 \%$ do total da amostra, participa com $5,13 \%$ do total de empresas que obtiveram faturamento com novos produtos no período considerado pela PAEP.

Já, como destaques negativos, é possível citar os setores de Vestuário e Acessórios, que, embora corresponda a 11,49\% do total de empresas da amostra, contribui com apenas $0,88 \%$ do total de empresas que obtiveram faturamento com novos produtos; e o de Alimentos e Bebidas, em que estes percentuais são respectivamente $9,92 \%$ e $5,07 \%$.

Um outro aspecto relevante para descrever a amostra utilizada pela PAEP é a sua distribuição com relação à faixa de pessoal ocupado (tamanho da empresa), em que é possível observar pela Figura 4 que sua composição apresenta a maior parcela de empresas na faixa de 5 a 29 pessoas ocupadas, correspondendo a $78,27 \%$ do total, a segunda maior parcela é de empresas na faixa de 30 a 99 pessoas ocupadas ou $14,05 \%$ do total da amostra, enquanto que a faixa de 100 a 499 corresponde a $6,06 \%$ e a faixa de 500 ou mais pessoas ocupadas corresponde a apenas $1,62 \%$.

Para as empresas que obtiveram faturamento com produtos novos, embora esta ordem se mantenha como era de se esperar, devido à distribuição de tamanho da amostra, percebe-se que o percentual de empresas com até 29 pessoas ocupadas diminui, enquanto que os percentuais das empresas nas faixas de 30 a 99 pessoas, 100 a 499 e 500 ou mais pessoas ocupadas aumentam acentuadamente, chegando a ser mais do que o dobro nas duas últimas faixas se comparado aos percentuais encontrados para as empresas da amostra.

Esse efeito pode ser explicado em decorrência das empresas maiores possuírem mais recursos para investir em pesquisa e desenvolvimento ou engenharia não rotineira, o que aumenta a possibilidade de resultados em termos de inovação tecnológica em produtos e, consequentemente, tende a aumentar o percentual de faturamento oriundo da venda destes itens, exceção feita a empresas de base tecnológica de pequeno ou médio porte.

Outros aspectos relevantes sobre a amostra estão presentes nas Figuras 5 e 6, que mostram a sua composição em termos de orientação exportadora e origem do capital controlador, respectivamente.

A grande maioria das empresas que compõem a amostra, segundo a Figura 5, é de empresas não exportadoras, representando $83,82 \%$ do total, enquanto que as empresas exportadoras correspondem a $10,96 \%$. Entretanto, ao se observar as empresas que obtiveram faturamento provindo de novos produtos no período considerado pela pesquisa, verifica-se o oposto, sendo que a maioria das empresas que obtiveram este resultado é exportadora (seja regularmente ou

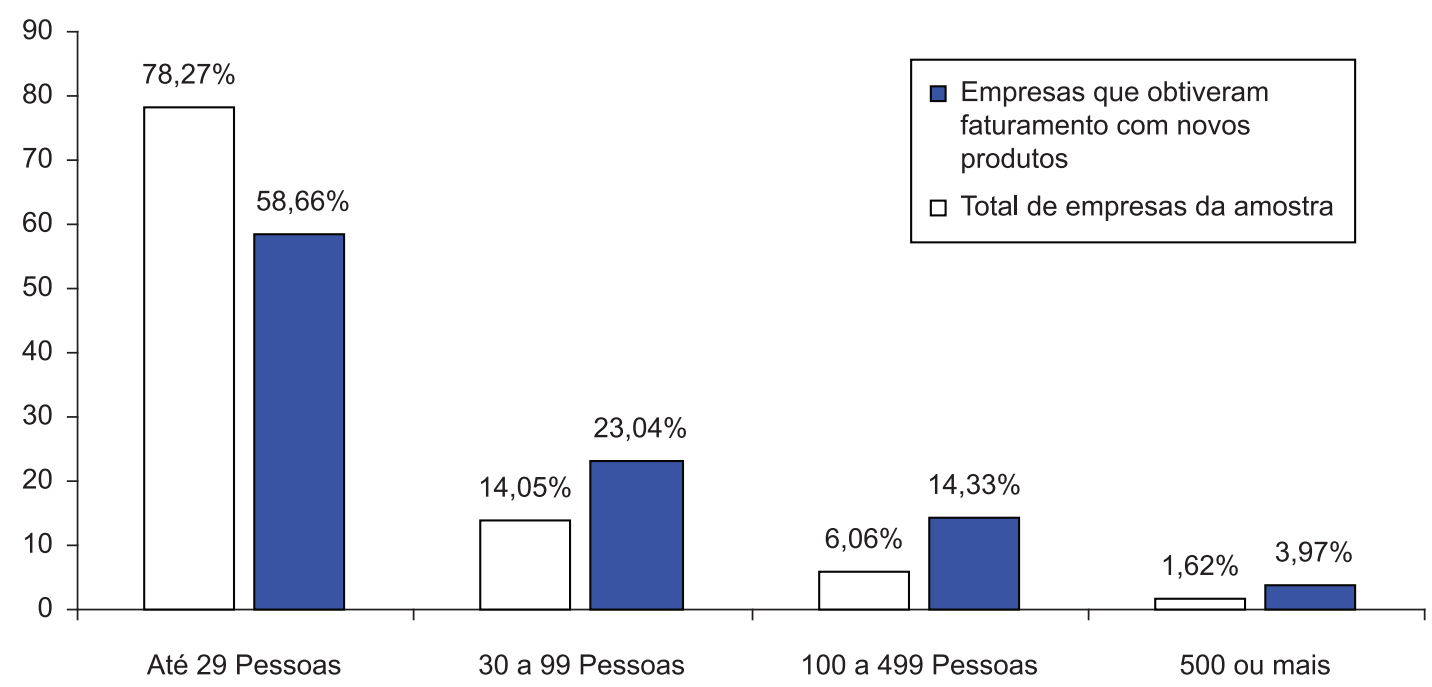

Figura 4. Distribuição percentual em relação ao tamanho das empresas. Fonte: Elaborado pelos autores. 
de maneira eventual), correspondendo a $67,41 \%$, enquanto que as não exportadoras correspondem a $31,17 \%$.

Os percentuais relacionados à orientação exportadora podem ser explicados pelo fato de que as empresas, ao introduzirem novos produtos em seu portfólio, tendem a conseguir diferenciação em relação aos concorrentes e, portanto, se tornam mais competitivas no exigente mercado externo.

Com relação à origem do capital controlador das empresas, os dados da Figura 6 mostram que a imensa maioria é de empresas cujo capital controlador é de origem nacional, sendo esta parcela de $92,83 \%$, contra $6,66 \%$ de empresas com capital controlador de origem internacional ou mista.

Considerando as empresas que obtiveram faturamento provindo de novos produtos, existe

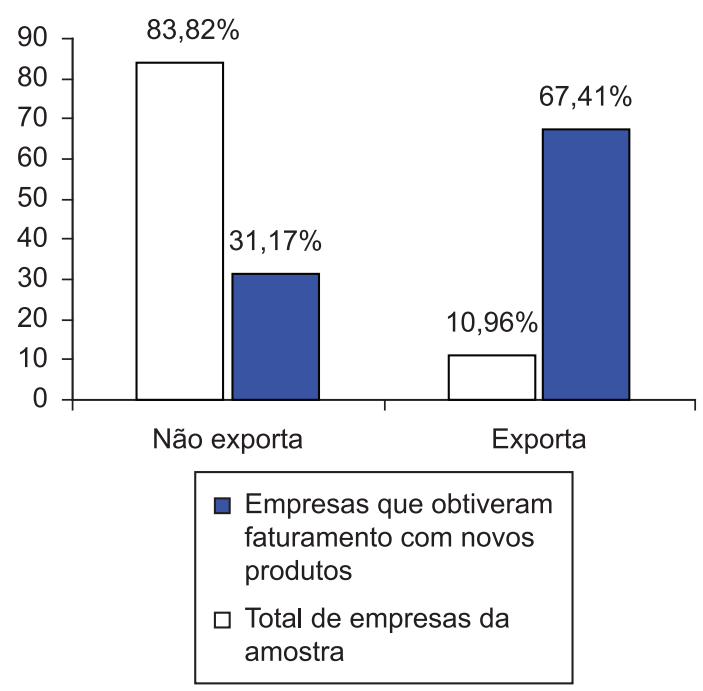

Figura 5. Orientação exportadora.

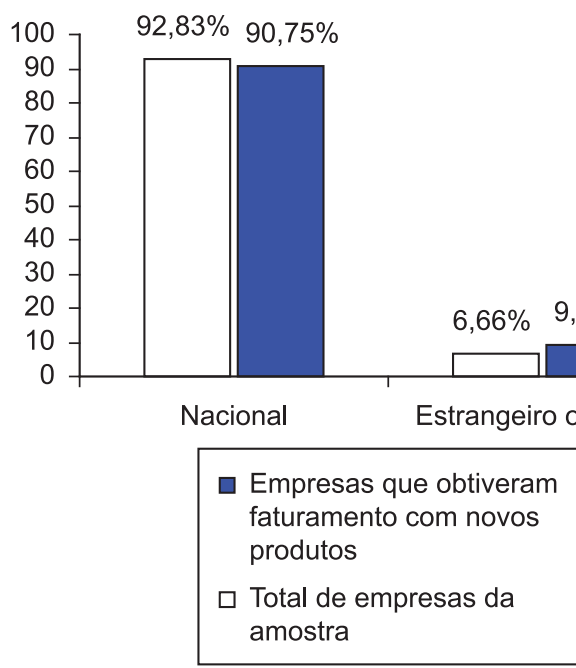

Figura 6. Origem do capital controlador. Fonte: Elaborado pelos autores. uma pequena variação na participação de empresas cujo capital controlador é de origem internacional ou mista, aumentando para $9,25 \%$, enquanto que a parcela de empresas com capital controlador nacional é de $90,75 \%$.

É preciso destacar que, em alguns casos a soma das parcelas não corresponde a $100 \%$, porque algumas empresas da amostra optaram por não responder a determinadas perguntas do questionário da PAEP.

Por meio dos dados apresentados nos gráficos, verifica-se que a obtenção de faturamento com novos produtos recebe certa influência do tamanho da empresa, da orientação exportadora e da origem do capital controlador, posto que os percentuais de empresas com tamanho superior, de empresas exportadoras e de empresas com capital controlador internacional ou misto são superiores aos das empresas que obtiveram receita provinda desta fonte. Cabe destacar que estes três fatores são citados amplamente na literatura como condicionantes do desempenho inovador das empresas.

\section{Apresentação e discussão dos resultados}

A técnica de regressão múltipla pôde ser utilizada neste estudo após a verificação dos pressupostos citados na seção 3. Para tanto, realizou-se inicialmente o teste de significância do modelo de regressão visando identificar o relacionamento entre a variável dependente e as variáveis independentes, utilizando o teste F (Fisher-Snedecor), cujo resultado foi de 3976,32, permitindo inferir que existe relação linear entre as variáveis dependentes e independentes.

Outro aspecto relevante para garantir o uso da técnica é a verificação de que os resíduos não estão autocorrelacionados, sendo utilizado o teste de Durbin-Watson que obteve como resultado o valor de 1,3821, que permite o emprego do método dos mínimos quadrados para o modelo de regressão múltipla utilizado.

Finalmente, o modelo foi testado quanto à multicolinearidade, sendo que o valor de VIF foi inferior a 0,4 para todas as variáveis, o que é considerado aceitável.

Os principais resultados encontrados pela pesquisa estão disponibilizados na Tabela 1, na qual é possível identificar as variáveis estatisticamente significativas para explicar o faturamento advindo de novos produtos (variáveis que possuem estatística t superior a 1,96 para níveis de significância iguais a 0,01 e 0,05). E um incremento nestas variáveis altera este faturamento conforme o coeficiente B da primeira coluna.

Ao analisar os resultados, verifica-se que o modelo possui um bom poder explicativo para a variável dependente em questão (obtenção de faturamento provindo de novos produtos). 
Sob este aspecto, Hair et al. (1998) afirmam que modelos de regressão com coeficientes $\mathrm{R}^{2}$ acima de 0,6 são considerados de alta acuracidade e o obtido foi 0,61 . Isso significa que as variáveis independentes conseguem explicar $61,57 \%$ da variância encontrada para a variável dependente, indicando que o modelo de pesquisa foi corretamente especificado.

Na Tabela 1, também se observa que a origem do capital controlador possui coeficiente que indica que as empresas com capital controlador de origem internacional possuem o faturamento provindo de novos produtos $4,74 \%$ maior do que empresas que possuem capital controlador de origem nacional. Este resultado corrobora o estudo de Kannebley Júnior, Porto e Pazzelo (2005), no qual se verificou que esta variável também estava associada a empresas inovativas no Brasil como um todo, de forma que a realidade do Estado de São Paulo está alinhada com relação a esta variável.

Os resultados também mostram que quando a empresa pertence a um grupo empresarial o percentual do faturamento com novos produtos aumenta em $13,71 \%$, o que pode ser explicado devido a esta relação empresarial favorecer a troca de conhecimento, investimentos e tecnologia entre as organizações do grupo, aumentando a possibilidade de ocorrer inovações tecnológicas. Esta variável foi discutida nos trabalhos de Mahmood e Lee (2004) e Hsieh, Yeh e Chen (2010), que serviram de referência para este estudo, em que ambos destacam a relevância da atuação em grupos empresariais e sua influência positiva no desempenho inovador das organizações investigadas. Neste estudo, embora sem a mesma intensidade que nas obras citadas, a influência desta variável no desempenho inovador também foi verificada, suportando os resultados anteriores apresentados na literatura.

Já a variável relativa aos recursos humanos alocados em atividades diretamente ligadas à produção mostra que um aumento de uma unidade diminui o faturamento provindo de novos produtos em $5,97 \%$, indicando que empresas cuja tendência seja alocar mais recursos humanos em suas plantas produtivas em detrimento de outras áreas (como pesquisa e desenvolvimento)

Tabela 1. Resultados da técnica de regressão linear múltipla.

\begin{tabular}{|c|c|c|c|c|c|}
\hline \multirow[t]{2}{*}{ Fatores de influência } & \multicolumn{2}{|c|}{ Coeficientes não padronizados } & \multirow{2}{*}{$\begin{array}{c}\begin{array}{c}\text { Coeficientes } \\
\text { padronizados }\end{array} \\
\text { B }\end{array}$} & \multirow[t]{2}{*}{$\mathbf{t}$} & \multirow[t]{2}{*}{ Significância } \\
\hline & B & Erro padrão & & & \\
\hline Tamanho & $-2,0585$ & 1,4933 & $-0,0386$ & $-1,3784$ & 0,1682 \\
\hline Orientação exportadora & $-0,4002$ & 1,4671 & $-0,0060$ & $-0,2727$ & 0,7851 \\
\hline $\begin{array}{l}\text { Origem do capital } \\
\text { controlador }\end{array}$ & 4,7407 & 2,2219 & 0,0439 & 2,1336 & $0,0330^{*}$ \\
\hline Salário médio & $-7,0355$ & 1,6783 & $-0,0884$ & $-4,1919$ & 0,8043 \\
\hline Idade da empresa & $-0,0154$ & 0,0408 & $-0,0074$ & $-0,3771$ & 0,7061 \\
\hline $\begin{array}{l}\text { Pertencer a grupo } \\
\text { empresarial }\end{array}$ & 13,7085 & 3,7617 & 0,0772 & 3,6442 & $0,0003 * *$ \\
\hline RH ligado à produção & $-5,9749$ & 1,5205 & $-0,0831$ & $-3,9295$ & $0,0001 * *$ \\
\hline Bens ou serviços & 31,2768 & 53,9732 & 0,0104 & 0,5795 & 0,5623 \\
\hline $\begin{array}{l}\text { Investimentos financeiros } \\
\text { em P\&D }\end{array}$ & 11,9307 & 2,8914 & 0,0621 & 0,05049 & 0,959765 \\
\hline RH em P\&D & 4,0470 & 4,8558 & 0,1762 & 8,2473 & $0,0000 * *$ \\
\hline Laboratório de P\&D & $-1,9476$ & 1,4352 & $-0,0279$ & $-1,3570$ & 0,1749 \\
\hline Cooperação em P\&D & 1,2688 & 1,6133 & 0,0150 & 0,7865 & 0,4317 \\
\hline Apoio Governamental & 5,8661 & 1,9580 & 0,0538 & 2,9961 & $0,0028 * *$ \\
\hline Fontes internas & 4,8279 & 1,2843 & 0,0727 & 3,7592 & $0,0002 * *$ \\
\hline Fontes ligadas ao mercado & 5,6886 & 1,3236 & 0,0836 & 4,2979 & $0,0000^{* *}$ \\
\hline Fontes acadêmicas & $-4,0408$ & 1,7915 & $-0,0435$ & $-2,2555$ & $0,0242 *$ \\
\hline Outras fontes & 0,1883 & 1,5699 & 0,0024 & 0,1199 & 0,9046 \\
\hline $\begin{array}{l}\text { Ampliação da variedade } \\
\text { de produtos }\end{array}$ & 1,2894 & 1,1651 & 0,0207 & 1,1067 & 0,2685 \\
\hline $\begin{array}{l}\text { Redução da variedade de } \\
\text { produtos }\end{array}$ & $-7,3624$ & 2,2123 & $-0,0976$ & $-3,3279$ & 0,0009 \\
\hline$*$ Significante a $5 \%$ & & $\mathrm{R}$ & $\mathrm{R}^{2}$ & $\mathrm{R}^{2}$ ajustado & Erro padrão \\
\hline$* *$ Significante a $1 \%$ & & 0,7847 & 0,6157 & 0,5531 & 19,3511 \\
\hline
\end{tabular}

Fonte: Elaborada pelos autores. 
têm menor probabilidade de inovar em produtos e, em decorrência disso, não obter este tipo de faturamento. Esta variável pode ser considerada uma variável proxy do tamanho das organizações, porém relacionada ao tamanho de suas atividades de transformação e operações produtivas. Por este prisma, verifica-se uma discrepância com os resultados do trabalho de Shefer e Fenkel (2005) e novamente com Kannebley Júnior, Porto e Pazzelo (2005) e outros estudos na literatura que usualmente apontam esta como uma característica das organizações inovadoras.

$\mathrm{O}$ resultado apresentado no parágrafo anterior pode apontar que não basta que a organização seja considerada grande se muitos de seus recursos humanos estiverem alocados em atividades de transformação. Pode ser preciso que uma parcela relevante destes recursos seja disponibilizada para atividades inovativas, caso a organização busque aumentar seu faturamento com novos produtos.

Desta forma, outro fator que impacta positivamente a variável dependente são os recursos humanos alocados em pesquisa e desenvolvimento; no caso deste trabalho, um incremento nesta variável indica que o percentual de faturamento provindo de novos produtos aumenta em $4,04 \%$, o que pode ser explicado pelo conhecimento gerado pela função $\mathrm{P} \& \mathrm{D}$ que tende a gerar inovações tecnológicas e, consequentemente, aumentar o percentual de faturamento com produtos novos. Esta variável está presente também nos trabalhos de Cooper, Edgett e Kleinshmidt (2005) e Millson e Wilemon (2006), cujos resultados indicam que empresas que possuem mais recursos para investir em $\mathrm{P} \& \mathrm{D}$ possuem maior probabilidade de inovar. Assim, este estudo confirma os resultados destes autores, mostrando a relevância destes investimentos para as indústrias paulistas.

$\mathrm{O}$ apoio governamental para atividades de inovação (financiamentos ou subsídios) também é estatisticamente significativo e causa um impacto positivo, da ordem de $5,87 \%$ sobre o faturamento provindo de novos produtos. Isto se explica devido ao fato de que estes recursos estimulam a empresa a realizar pesquisa e desenvolvimento, gerando inovações em produtos e aumentando o percentual desta variável. Verifica-se que este resultado alinha-se ao de outros estudos presentes na literatura, como os de Sharp e Pavitt (1993), Fonseca (2001) e Freitas e Tunzelmann (2008), nos quais a presença do apoio governamental é de influência relevante no desempenho inovador das organizações, o que se confirma nesta pesquisa no que se refere ao faturamento provindo de novos produtos.

Também as fontes de informação para atividades inovativas são estatisticamente significativas para explicar o faturamento provindo de novos produtos na indústria. Assim, o coeficiente da variável relacionada com as fontes internas (departamento de P\&D ou outras áreas da empresa) mostra que atribuir importância a este tipo de fonte aumenta o faturamento em 4,83\%, o que pode ser explicado pela alta aplicabilidade do conhecimento gerado por estas fontes em inovações tecnológicas em produtos, o que, por sua vez, pode implicar em um aumento do percentual de faturamento provindo da venda destes itens.

O coeficiente da variável relativa às fontes ligadas ao mercado mostra que a atribuição de importância a estes tipos de fontes causa um aumento no faturamento provindo de novos produtos em 5,69\%, o que pode ser explicado pelo caráter comercial do conhecimento provindo de consumidores, fornecedores e concorrentes, o que ajuda a empresa a aumentar as vendas destes produtos.

Já as fontes acadêmicas também apresentam significância estatística, sendo que a atribuição de importância a estas fontes tem impacto negativo, diminuindo o faturamento provindo de novos produtos em $4,04 \%$, possivelmente refletindo o caráter pouco comercial em termos de inovação em produtos do conhecimento gerado nestas instituições, ou ainda, que as empresas não buscam este tipo de fonte de informação quando necessitam de apoio para as atividades de inovação, como as ligadas ao mercado que são mais procuradas, havendo uma perda de potencial acadêmico que não é utilizado pelas empresas.

Todos os tipos de fontes de informações para inovação citados acima foram discutidos no estudo de Quadros et al. (2001), cujas conclusões destacam sua relevância para explicar o desempenho inovador da indústria paulista em detrimento de atividades internas de P\&D. Também deve-se ressaltar que, quanto às fontes acadêmicas e ligadas ao mercado, os resultados encontrados neste trabalho alinham-se parcialmente ao estudo de Frishammar e Hörte (2005), que afirmam que organizações que gerenciam melhor as informações provindas destas fontes possuem melhor desempenho inovador, o que não se confirma no que tange às fontes acadêmicas.

Por fim, cabe ressaltar que algumas variáveis que poderiam ser inicialmente consideradas relevantes para explicar o faturamento com novos produtos como as associadas à gestão de portfólio (aumento e redução do número de produtos) e algumas associadas à $\mathrm{P} \& \mathrm{D}$ (cooperação em $\mathrm{P} \& \mathrm{D}$, recursos financeiros alocados em P\&D e laboratório ou departamento específico de $P \& D$ ) não foram consideradas significativas neste estudo, o que pode refletir o baixíssimo número de organizações que possuem estas características, o que é perceptível quando se observam os resultados da PAEP.

\section{Considerações finais}

Os resultados encontrados por este trabalho permitem concluir que a obtenção de faturamento com novos produtos pela indústria do Estado de São Paulo pode ser explicada pelas variáveis presentes no modelo conceitual elaborado para este fim. Assim, 
foi possível observar que os fatores que influenciam este fenômeno são: a origem do capital controlador; a empresa pertencer a grupo empresarial; o percentual de recursos humanos alocados em atividades diretamente ligadas à produção; o percentual de recursos humanos alocados em P\&D; o apoio governamental para atividades inovativas; e a atribuição de importância para as fontes de informação internas (departamento de $P \& D$ e outros departamentos), fontes de informação ligadas ao mercado (fornecedores, consumidores e concorrentes) e as chamadas fontes acadêmicas (universidades e centros de pesquisa).

Entre os fatores de influência encontrados, todos aumentam o faturamento provindo de novos produtos, com exceção do percentual de recursos humanos alocados em atividades diretamente ligados à produção, o que, de certa forma, é um resultado esperado, já que empresas que inovam e que consequentemente obtêm melhores resultados com a venda de novos produtos tendem a alocar menos recursos nestas atividades e mais em atividades como pesquisa e desenvolvimento e engenharia não rotineira; e também com exceção da atribuição de importância às fontes de informação acadêmicas, que, como já discutido, pode estar relacionado ao caráter pouco comercial do conhecimento gerado por estas fontes.

De modo geral, os resultados encontrados neste estudo corroboram alguns resultados obtidos por estudos presentes na literatura, como já destacado anteriormente, porém com exceção das variáveis de RH ligado à produção e Fontes acadêmicas que, embora citadas na literatura como influências positivas no desempenho inovativo das firmas, apresentaram influência oposta neste estudo. Além disso, diversos fatores citados na literatura não apresentaram significância para a indústria paulista (como pode ser observado na Tabela 1) indicando que o seu perfil inovativo apresenta peculiaridades, pelo menos quando se toma por proxy de inovação a variável faturamento advindo de novos produtos.

Estes resultados indicam que empresas industriais do Estado que tenham a intenção de aumentar o faturamento com produtos novos devem dar atenção aos fatores aqui citados, buscando incorporá-los em seus modelos de decisão e suas estratégias empresariais de forma a maximizar sua influência sobre o fenômeno estudado.

Como principal limitação da pesquisa é possível destacar a não inclusão de todos os fatores de influência do fenômeno no modelo conceitual, fato que se deve a própria natureza de qualquer modelo por se tratar de uma representação teórica e simplificada da realidade.

Outro aspecto que pode ser citado dentro deste contexto é o período de coleta de dados (1999-2000), que poderia refletir uma realidade mais atual, porém destaca-se que a PAEP é uma pesquisa de grande amplitude, validade e relevância e sem similar no que se refere à indústria paulista, o que compensa o fato de não haver ainda uma versão sobre um período mais atual, além disso, quando se observam os dados referentes à indústria paulista na PINTEC, verificam-se poucas mudanças no que se refere à contribuição econômica e tecnológica do Estado nas suas três versões, o que indica que os dados colhidos pela PAEP ainda podem ser utilizados.

Como sugestões para trabalhos futuros, destacam-se a identificação e incorporação de novos fatores ao modelo proposto e também a realização de estudos setoriais para o entendimento das peculiaridades de cada setor industrial, de forma a investigar aspectos que não foram aqui discutidos.

Espera-se que este trabalho possa contribuir para o entendimento de como os fatores estudados podem influenciar o faturamento provindo de novos produtos nas indústrias do Estado de São Paulo, servindo de referência para novos estudos nesta área, ou para a definição de estratégias empresariais relacionadas ao tema.

\section{Referências}

ARCHIBUGI, A.; HOWELLS, J.; MICHIE, J. Innovation systems in a global economy. Technology Analysis \& Strategic Management, v. 11, n. 4, p. 527-539, 1999. http://dx.doi.org/10.1080/095373299107311

BAKER, W. E.; SINKULA, J. M. Market Orientation and the New Product Paradox. Journal of Product Innovation Management, v. 22, n. 4, p. 483-502, 2005. http://dx.doi.org/10.1111/j.1540-5885.2005.00145.x

BUSH, V. Science - The Endless Frontier: A Report to the President on a program for Postwar scientific Research. Washington: Office of Scientific Research and Development, 1945.

CARAYANNIS, E. G.; ROY, R. I. S. Davids vs Goliaths in the small satellite industry: the role of technological innovation dynamics in firm competitiveness. Technovation, v. 20, n. 6, p. 287-297, 2000. http:// dx.doi.org/10.1016/S0166-4972(99)00137-6

CASTELLACCI, F. Technological Paradigms, Regimes and Trajectories: Manufacturing and Service Industries in a New Taxonomy of Sectoral Patterns of Innovation. Research Policy, v. 37, p. 978-994, 2008. http://dx.doi. org/10.1016/j.respol.2008.03.011

COOPER, R. G.; EDGETT, S. J.; KLEINSHMIDT, E. J. Benchmarking bets NPD practices - III. Research Technology Management, v. 47, n. 6, p. 43-55, 2005.

DAMANPOUR, F.; SCHNEIDER, M. Phases of the Adoption of Innovation in Organizations: Effects of Environment, Organization and Top Managers. British Journal of Management, v. 17, n. 3, p. 215-236, 2006. http://dx.doi.org/10.1111/j.1467-8551.2006.00498.x

DOSI, G. Sources, procedures and microeconomic effects of innovation. Journal of Economic Literature, v. 16, n. 3, p. 1120-1171, 1988.

FONSECA, R. Inovação tecnológica e o papel do governo. Brasília: Ministério da Ciência e Tecnologia, Centro de Estudos Estratégicos, 2001. (Parcerias Estratégicas, n. 13). 
FRANCIS, D.; BESSANT, J. Targeting innovation and implications for capability development. Technovation, v. 25, n. 3, p. 171-183, 2005. http://dx.doi.org/10.1016/j. technovation.2004.03.004

FREEL, M. S.; ROBSON, P. J. A. Small firm innovation, growth and performance - evidence from Scotland and northern England. International Small Business Journal, v. 22, n. 6, p. 561-575, 2004. http://dx.doi. org/10.1177/0266242604047410

FREITAS, I. M. B.; TUNZELMAN, N. Mapping Public support for innovation in a three-dimensional space. Research Policy, v. 37, p. 1446-1464, 2008.

FRISHAMMAR, J.; HÖRTE, S. A. Managing External Information in Manufacturing Firms: The Impact on Innovation Performance. The Journal of Product Innovation Management, v. 22, n. 3, p. 251-266, 2005. http://dx.doi.org/10.1111/j.0737-6782.2005.00121.x

GARSON, G. D. Factor Analysis. Quantitative Research in Public Administration, NC State University, 2008. Disponível em: <http://www2.chass.ncsu.edu/garson/ pa765/factor.htm>. Acesso em: 10 nov. 2008.

GREENE, W. H. Econometric analysis. 2nd ed. Englewood Cliffs: Prentice Hall, 1993.

GUJARATI, D. N. Econometria Básica. 3. ed. São Paulo: Makron Books, 2000.

HAGEDOORN, J. Measuring innovative performance: is there an advantage in using multiple indicators? Research Policy, v. 32, n. 8, p. 1365-1379, 2002. http://dx.doi. org/10.1016/S0048-7333(02)00137-3

HAIR, J. F. et al. Multivariate Data Analysis. 5th ed. New Jersey: Prentice Hall, 1998.

HALL, L. A.; BAGCHI-SEN, S. A study of P\&D, innovation, and business performance in the Canadian biotechnology industry. Technovation, v. 22, p. 231-244, 2002. http:// dx.doi.org/10.1016/S0166-4972(01)00016-5

HSIEH, T. J.; YEH, R. S.; CHEN, Y. J. Business Group characteristics and affiliated firm innovation: The case of Taiwan. Industrial Marketing Management, v. 39, n. 4, p. 560-570, 2010. http://dx.doi.org/10.1016/j. indmarman.2008.12.018

JOHNSON, R. A.; WICHERN, D. W. Applied multivariate statistical analysis. 3rd ed. New Jersey: Prentice Hall, 1992.

KANNEBLEY JÚNIOR, S.; PORTO, G. S.; PAZELLO, E. T. Characteristics of Brazilian innovative firms: An empirical analysis based on PINTEC- industrial research on technological innovation. Research Policy, v. 34, n. 6, p. 872-893, 2005. http://dx.doi.org/10.1016/j. respol.2005.04.003

KLINE, S. J.; ROSENBERG, N. An overview of innovation. In: LANDAU, R.; ROSENBERG, N. (Eds.). The
Positive Sum Strategy: Harnessing Technology for Economic Growth. Washington: National Academy Press, 1986. p. 275-305.

MADDALA, G. S. Introduction to Econometrics. 2nd ed. New Jersey: Prentice Hall, 1992.

MAHMOOD, I. P.; LEE, C. Y. Business groups: entry barrier innovation debate revisited. Journal of Economic Behavior and Organization, v. 54, n. 4, p. 513-531, 2004. http://dx.doi.org/10.1016/j. jebo.2002.12.003

MALHOTRA, N. K. Pesquisa de marketing: uma orientação aplicada. 3. ed. Porto Alegre: Bookman, 2001.

MANSURY, M. A.; LOVE, J. H. Innovation, productivity and growth in US business services: A firm-level analysis. Technovation, v. 28, n. 1-2, p. 52-62, 2008. http://dx.doi.org/10.1016/j.technovation.2007.06.002

MILLSON, M. R.; WILEMON, D. Driving new product success in the electrical equipment manufacturing industry. Technovation, v. 26, n. 11, p. 1268-1286, 2006. http://dx.doi.org/10.1016/j.technovation.2005.08.008

NUCHERA, A. H.; SERRANO, G. L.; MOROTE, J. P. La gestión de la innovación y la tecnologia en las organizaciones. Madrid: Ediciones Pirâmide, 2002.

ORGANISATION FOR ECONOMIC CO-OPERATION AND DEVELOPMENT - OCDE. Proposed Guidelines for Collecting and Interpreting Technological Innovation Data: Oslo Manual. Paris, 2005. 136 p. Disponível em: <http://www.oecd.org>. Acesso em: 14 mar. 2007.

QUADROS, R. et al. Technological innovation in Brazilian industry: an assessment based on the São Paulo innovation survey. Technological forecasting and social change, v. 67, n. 5, p. 203-219, 2001. http://dx.doi.org/10.1016/ S0040-1625(00)00123-2

SCHUMPETER, J. A. Essays on entrepreneurs, Innovations, Business Cycles, and the evolution of capitalism. New Brunswick: Transaction, 1991.

SHAPIRO, A. R. Measuring Innovation: Beyond Revenue from New Products. Research Technology Management, v. 49, n. 6, p. 42-51, 2006.

SHARP, M.; PAVITT, K. Technology Policy in the 1990s: Old Trends and New Realities. Journal of Common Market Studies, v. 31, n. 2, 1993. http://dx.doi. org/10.1111/j.1468-5965.1993.tb00454.x

SHEFER, D.; FRENKEL, A. R\&D, firm size and innovation: an empirical analysis. Technovation, v. 25, n. 1, p. 25-32, 2005. http://dx.doi.org/10.1016/ S0166-4972(03)00152-4

VIOTTI, E. B.; MACEDO, M. M. (Orgs.). Indicadores de ciência, tecnologia e inovação no Brasil. Campinas: Editora Unicamp, 2001. 